\title{
Transmission Line Based Wideband Microwave Sensor for Determination of Biodiesel Purification
}

\author{
Nutdechatorn Puangngernmak $^{1, \mathrm{a}}$, Kanokwan Sroykesorn ${ }^{2, \mathrm{~b}}$, Tawiwan Kangsadan ${ }^{2, \mathrm{c}}$, \\ and Suramate Chalermwisutkul ${ }^{1, \mathrm{~d}, *}$ \\ 1 RF and Microwave Laboratory, The Sirindhorn International Thai-German Graduate School of \\ Engineering, King Mongkut's University of Technology North Bangkok, Bangkok 10800, Thailand \\ 2 Chemical and Process Engineering Laboratory, The Sirindhorn International Thai-German Graduate \\ School of Engineering, King Mongkut's University of Technology North Bangkok, Bangkok 10800, \\ Thailand \\ E-mail: anutdechatorn@gmail.com, bk.sroykesorn@gmail.com, ctawiwan.k.cpe@tggs-bangkok.org, \\ dsuramate.c.ce@tggs-bangkok.org (Corresponding author)
}

\begin{abstract}
The main objective of this study is the application of the microwave measurement technique to determine electrical characteristics of material with complex permittivity values in a frequency range from $100 \mathrm{MHz}-10 \mathrm{GHz}$. The measurement results represent the purification level of biodiesel compared to the results analyzed by Gas Chromatography (GC) technique (modified EN14103). The microwave sensor was created based on a simple strip line structure of a microwave transmission line which is filled with the liquid under test (LUT) samples. Scattering parameters have been measured using a vector network analyzer (VNA). After that, the complex permittivity values and impulse response using inverse Fourier transformation of the propagation coefficient have been calculated and compared with the results from GC analysis. The results of complex permittivity can be used to describe the behavior of LUT in terms of relative permittivity related to the stored energy and the dissipation of energy within the sample. The levels of purification of biodiesel are represented by the relative complex permittivity of the LUT.

Another way to identify the level of purification uses the complex value of inverse Fourier transformation of the propagation coefficient. By doing this, the stored energy and the dissipation of the LUT can also be analyzed in time domain. Four biodiesel samples have been analyzed using the proposed sensor. The purification level can be obviously identified especially in a frequency range from 2 to $4 \mathrm{GHz}$.
\end{abstract}

Keywords: Material characterization, microwave sensor, biodiesel purification.

ENGINEERING JOURNAL Volume 19 Issue 5

Received 12 September 2014

Accepted 23 February 2015

Published 31 October 2015

Online at http://www.engj.org/

DOI:10.4186/ej.2015.19.5.29 


\section{Introduction}

The purification of biodiesel is one of several factors to determine the quality of biodiesel products. In an industrial chemical process, the purification of biodiesel products is analyzed by the Gas Chromatography (GC) method (modified EN14103) [1-2] which requires costly instruments [3]. The microwave measurement is another technique to characterize properties of material with a complex permittivity value which describes behavior of materials in the terms of stored and dissipated energies [4-5]. A dielectric permittivity spectrum over a wide range of frequency is not constant. It depends on electrical properties, ionic and dipolar relaxation and atomic and electronic resonance of the material which are affected by temperature and frequency of the electric field. In a high frequency measurement, the permittivity values cannot be directly measured, but are calculated from scattering parameters [6-8]. Scattering parameters can be obtained from microwave measurement using a vector network analyzer (VNA), a microwave sensor and high frequency cables.

In order to measure dielectric properties of liquid-based materials, various sensor type and measurement techniques have been proposed in the literature [9-10]. Prominent methods are based on transmission line theory [11] or reflection/transmission in a waveguide [12-13]. However, the accuracy is limited especially in case of material with low dielectric constant and loss tangent [15]. Another techniques for liquid characterization is the cavity perturbation method based on a two port transmission/reflection measurement, this method provides high accuracy of the determined dielectric properties, whereas the main drawback is a limited bandwidth [16-18].

The sensor proposed in this work was designed based on a transmission line model. The frequency range of the measurement was from $100 \mathrm{MHz}$ to $10 \mathrm{GHz}$. The wide frequency band of investigation was chosen in order to determine the most significant frequency range for the sensing purpose of an unknown substance in the liquid under test. In fact, the wider the bandwidth, the more information about the content of the liquid under test can be obtained. As an application example, the proposed sensor and a VNA have been utilized in an experiment to determine the purification in percent of four biodiesel samples. The samples of biodiesel product were prepared by the Chemical and Process Engineering Laboratory at the Sirindhorn International Thai-German Graduate School of Engineering (TGGS), whereas the microwave measurement was carried out at the TGGS RF and Microwave Laboratory. The results of the calculation with four samples have been compared with the GC analysis. The purification levels of $75.48,83.19,86.76$ and 96.89 percent have been investigated.

\section{Microwave Sensor Model and Equations}

The microwave sensor for the purification of biodiesel was designed based on a strip line model which is filled with the liquid under test (LUT) as the filling of the strip line. Geometries of the strip line are shown in Fig. 1. The model of the sensor is represented by a circuit model of a transmission line represented by series resistor $(R)$, shunt conductor $(G)$, shunt capacitor $(C)$ and series inductor $(L)$ [19] as shown in Fig. 2.

The ratio of the amplitude of voltage and current of a single wave propagating along the line is the characteristic impedance which is calculated by the transmission line model. The characteristic impedances of the transmission line and strip line models are given by Eq. (1) and (2) [20]. The characteristic impedance value is varied by the permittivity of the LUT contained as the filling of the strip line structure.

The sensor for biodiesel purification was designed using the optimal dimensions which provide the characteristic impedance of $5 \mathrm{O} / \sqrt{\epsilon_{\mathrm{r}}} \mathrm{Ohm}$. In case of air-filled strip line, the characteristic impedance is 50 $\mathrm{Ohm}$, for example. A picture of the sensor prototype is shown in Fig. 3.

$$
\begin{gathered}
Z_{c}=\frac{\sqrt{R+j \omega L}}{\sqrt{G+j \omega C}} \\
Z_{c}=\frac{30}{\sqrt{\varepsilon_{r}}} \ln \left\{1+\frac{A}{2}\left[A+\sqrt{A^{2}+6.27}\right]\right\}
\end{gathered}
$$


with

$$
A=\frac{16}{\pi\left(W^{\prime} / h\right)}, \frac{W^{\prime}}{h}=\frac{W}{h}+\frac{\Delta W}{h}, \frac{\Delta W}{h}=\left(\frac{t}{h}\right) \frac{1}{\pi}\left\{\left[\frac{1}{\frac{4 h}{t}+1}\right]^{2}+\left[\frac{1 / 4 \pi}{\frac{W}{t}+1.10}\right]^{\frac{6}{3+t / h}}\right\} .
$$

Considering electromagnetic wave transmitting through and reflected from a sample surrounding a transmission line as shown in Fig. 4, the reflection and transmission coefficient are expressed by scattering parameters, $S_{11}$ and $S_{21}$, as described in Eq. (3) and (4). It can also be shown that $S_{11}$ and $S_{21}$ are related to the reflection and transmission coefficients by the Eq. (5) and (6).

$$
\Gamma=K \pm \sqrt{K^{2}-1}
$$

with $K=\frac{\left(S_{11}^{2}-S_{21}{ }^{2}\right)+1}{2 \times S_{11}}$.

$$
\begin{gathered}
T=\frac{\left(S_{11}+S_{21}\right)-\Gamma}{1-\left(S_{11}+S_{21}\right) \Gamma} \\
S_{11}=\frac{\Gamma\left(1-T^{2}\right)}{1-\Gamma^{2} T^{2}} \\
S_{21}=\frac{T\left(1-\Gamma^{2}\right)}{1-\Gamma^{2} T^{2}}
\end{gathered}
$$

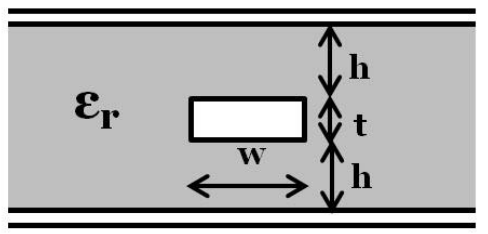

Fig.1. Strip line geometries.

Fig. 2. Equivalent circuit model of a transmission line.
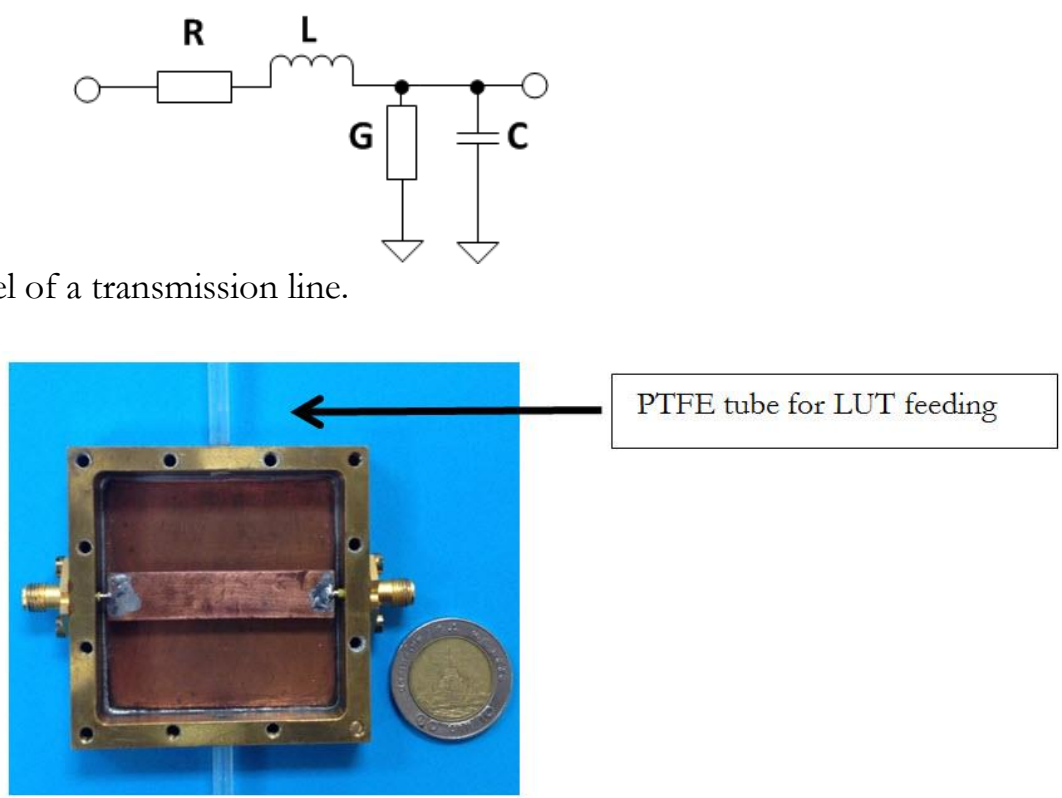

Fig. 3. The sensor prototype when the top cover is removed. 


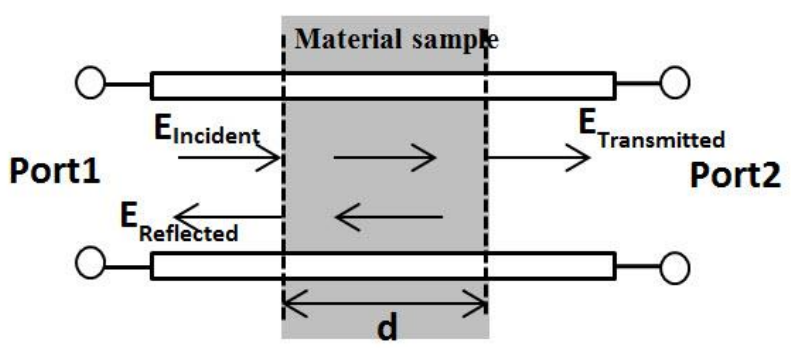

Fig. 4. Electromagnetic wave transmitting through and reflected from a sample in a transmission line.

\section{Measurement Setup}

The measured scattering parameters are provided by a VNA. The measurement setup is shown in Fig. 5 consisting of the microwave sensor prototype, high frequency cables and a VNA. The frequency was swept from $100 \mathrm{MHz}$ to $10 \mathrm{GHz}$. The LUT is fed into the sensor by hypodermic syringe and a flexible rubber tube. After finishing each sample, the sensor must be cleaned by feeding distilled water into the sensor and draining it out before a new measurement can be performed. Four LUT samples of biodiesel products with different purification levels of 75.48, 83.19, 86.76 and 96.89 percent have been investigated.

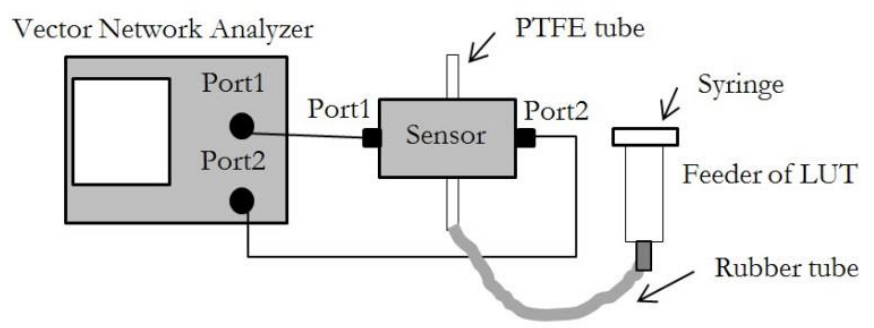

Fig. 5. Measurement setup for determination of biodiesel purification level.

\section{Calculation of the Permittivity and Time Domain Analysis}

The reflection and transmission coefficients of the sensor are related to electromagnetic wave transmitting through and reflected from a sample filled in the transmission line configuration as shown in Fig. 4. The reflection coefficient is given by

$$
\Gamma=\frac{Z-Z_{0}}{Z+Z_{0}}=\frac{\sqrt{\mu_{r} / \varepsilon_{r}}-1}{\sqrt{\mu_{r} / \varepsilon_{r}}+1}
$$

where $\varepsilon_{r}$ and $\mu_{r}$ are relative permittivity and permeability of LUT, respectively.

The transmission coefficient is

$$
T=\exp (-j \omega \sqrt{\mu \varepsilon} \times d)=\exp \left(-j\left(\omega / c_{0}\right) \sqrt{\mu_{r} \varepsilon_{r}} d\right)
$$

where $d$ is the length of strip line surrounded by the LUT and $c_{o}$ is the speed of light.

Define

$$
\sqrt{\mu_{r} \varepsilon_{r}}=A+j B \text { and } \frac{d}{c_{0}}=k
$$

then

$$
T=\exp (-k B \omega)[\cos (k A \omega)+j \sin (k A \omega)]
$$

Consider the real part of the transmission coefficient

$$
T_{1}=\operatorname{Re} a l(T)=\exp (-k B \omega)[\cos (k A \omega)]
$$


Then, consider minima and maxima of $T 1$ to solve $A_{n_{-} \text {Min }}$ and $A_{n_{-} \operatorname{Max}}$ where $n_{-}$Min and $n_{-}$Max of $A_{n_{-} \text {Min }}$ and $A_{n_{-} \text {Max }}$ are indices for the case of the minima and maxima of $T 1, f_{n_{-} \text {Min }}$ and $f_{n_{-} \text {Max }}$ is the frequency at the minima and maxima.

$$
\begin{gathered}
A_{n_{-} \operatorname{Min}}=\left(\frac{1}{2}\right) \frac{(2 n)-1}{k f_{n_{-} \operatorname{Min}}} \\
A_{n_{-} \operatorname{Max}}=\frac{n}{k f_{n_{-} \operatorname{Max}}}
\end{gathered}
$$

Consider minima and maxima of $T 1$ to solve for $B_{n_{-} \operatorname{Min}}$ and $B_{n_{-}} \operatorname{Max}$ where $n_{-}$Min and $n_{-}$Max are indices for the case of the minima and maxima of $T 1$

$$
\begin{gathered}
\frac{T 1_{n_{-} \text {Min }}}{T 1_{n_{-} \text {Min }+1}}=\exp \left[-k B_{n_{-} \min }\left(\omega_{n_{-} \text {Min }}-\omega_{n_{-} \text {Min }+1}\right)\right] \\
B_{n_{-} \text {Min }}=\left(\frac{1}{k}\right) \ln \left(\frac{T 1_{n_{-} \text {Min }}}{T 1_{n_{-} \text {Min }+1}}\right)\left(\frac{1}{2 \pi\left(f_{n_{-} \text {Min }+1}-f_{n_{-} \text {Min }}\right)}\right) \\
B_{n_{-} \text {Max }}=\left(\frac{1}{k}\right) \ln \left(\frac{T 1_{n_{-} \text {Max }}}{T 1_{n_{-} \text {Max }+1}}\right)\left(\frac{1}{2 \pi\left(f_{n_{-} \text {Max }+1}-f_{n_{-} \text {Max }}\right)}\right)
\end{gathered}
$$

In case of non-ferrous metal $\mu_{r}=1$, from $\sqrt{\mu_{r} \varepsilon_{r}}=A+j B$ then

$$
\varepsilon_{r}=\varepsilon^{\prime}-j \varepsilon^{\prime \prime}=\left(A^{2}-B^{2}\right)-j(2 A B)
$$

An alternative way for the characterization can be provided by applying Fourier transformation (FT) to the real part of the transmission coefficient. The wide frequency band of investigation is required in order to obtain a sufficient resolution of the Fourier transformation. The frequency domain is then transformed into another fictitious domain which represents significant difference among the LUTs with different purification. The FT equation are given by

$$
\begin{gathered}
T 1_{(\omega)}=\exp (-k B \omega)[\cos (k A \omega)] \\
F T\left(T 1_{(\omega)}\right)=T 1_{(\tau)}=\frac{1}{2 \pi} \int_{-\infty}^{\infty} \exp (-k B \omega)[\cos (k A \omega)] \exp (-j \omega \tau) \cdot \partial \omega \\
F T\left(T 1_{(\omega)}\right)=T 1_{(\tau)}=\left(\frac{1}{2}\right)\left[\frac{(k B)+j(k A-\tau)}{(k B)^{2}+(k A-\tau)^{2}}\right]
\end{gathered}
$$

In another way, the inverse Fourier transformation (IFT) into time ( $\mathrm{t}$ ) domain can also be performed (see Eq. (21)). The IFT result (see Eq. (22)) is the complex conjugate of the FT result (see Eq. (19) and (20)).

$$
\begin{gathered}
\operatorname{IFT}\left(T 1_{(\omega)}\right)=T 1_{(t)}=\frac{1}{2 \pi} \int_{-\infty}^{\infty} \exp (-k B \omega)[\cos (k A \omega)] \exp (j \omega t) \cdot \partial \omega \\
\operatorname{IFT}\left(T 1_{(\omega)}\right)=T 1_{(t)}=\left(\frac{1}{2}\right)\left[\frac{(k B)-j(k A-\tau)}{(k B)^{2}+(k A-\tau)^{2}}\right]
\end{gathered}
$$

\section{Simulation and Measurement Results}

After conceptual design of the sensor with mathematical derivation in previous section, $\mathrm{S}_{21}$ of the sensor have been simulated using CST Microwave Studio for cases with various filling materials. The real part of $\mathrm{S}_{21}\left(\operatorname{Re}\left\{\mathrm{S}_{21}\right\}\right)$ is then used for further analysis of the material property. 


\subsection{Simulation Results}

The model of microwave sensor was optimized, created and simulated by CST. The space around the strip is filled by CST models of material which have different permittivity values. Three material models which are used in this simulation are air, water (first order Debye model) and a fictive dielectric material (dielectric constant $=3$, tangent delta $=0.1$ at $10 \mathrm{GHz}$ (first order Debye model [21]). The simulated real parts of $\mathrm{S}_{21}$ in case of all filling materials in a frequency range from $100 \mathrm{MHz}$ to $10 \mathrm{GHz}$ are show in Fig. 6.

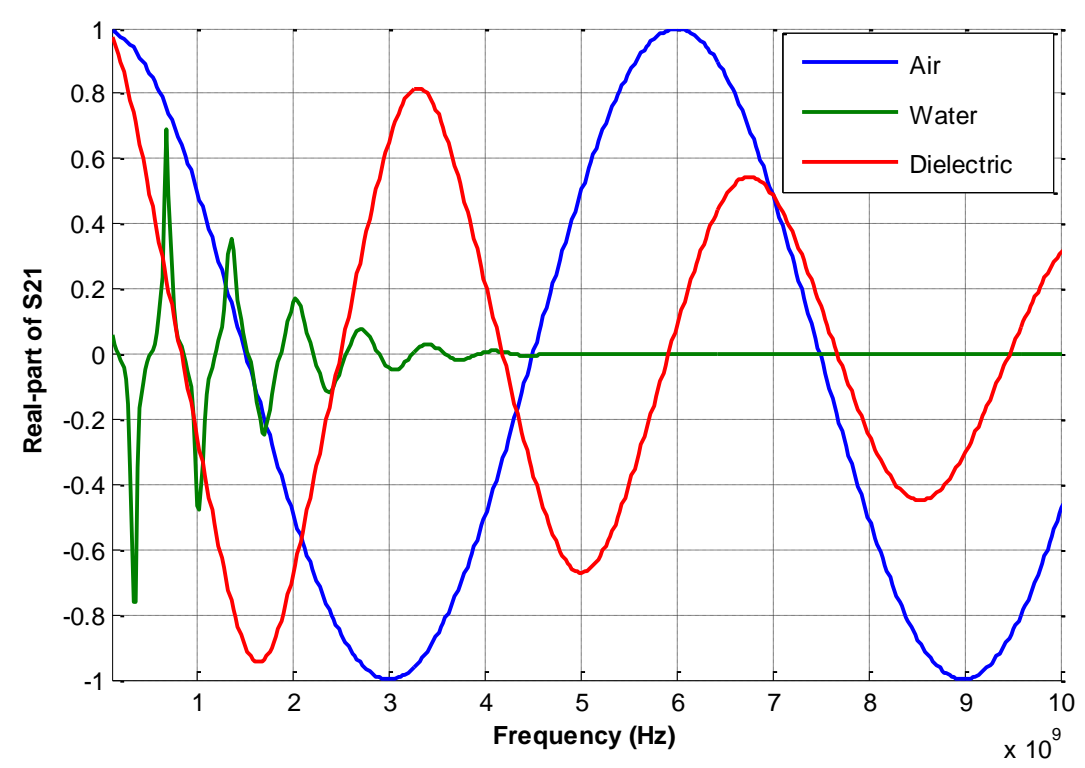

Fig. 6. Real part of $S_{21}$ with various filling materials.

From the Fig. 6, the dielectric constant and loss tangent of air are one and zero, respectively. For the Debye model of water, the dielectric constant is 78.4 at static electric field and the loss tangent is 0.9 at 10 $\mathrm{GHz}$ The amplitude of $\operatorname{Re}\left\{\mathrm{S}_{21}\right\}$ decreases with increased loss tangent of the material. The frequency difference between neighboring zero-crossing points decreases with increased dielectric constant of the sample.

\subsection{Measurement and Calculation Results}

Biodiesel samples with purification levels of $75.48,83.19,86.76$ and 96.89 percent were filled in the strip line sensor. The scattering parameters, $S_{11}$ and $S_{21}$, were measured ten times for each sample. The measurement results of the $S_{11}$ magnitudes for the samples with 75.48, 86.76 and 96.89 percent purification are shown in Fig.7. The result of the 83.19 percent was intentionally left out because it cannot be well separated from the result with 86.76 percent. However, by zooming into a frequency range from 3 to 3.5 $\mathrm{GHz}$ as shown in Fig.8, the result with 83.19 percent can be well recognized to be different in comparison to the other purification levels. Similarly, the difference of $S_{21}$ between 83.19 and 86.76 is also not significant. So, $\mathrm{S}_{21}$ in case of $75.48,86.76$ and 96.89 percent of purification are plotted in Fig. 9. In addition, because $S_{11}$ is negligible, the transmission coefficient ( $T$ ) in Eq. (6) is approximately equal to $S_{21}$. 


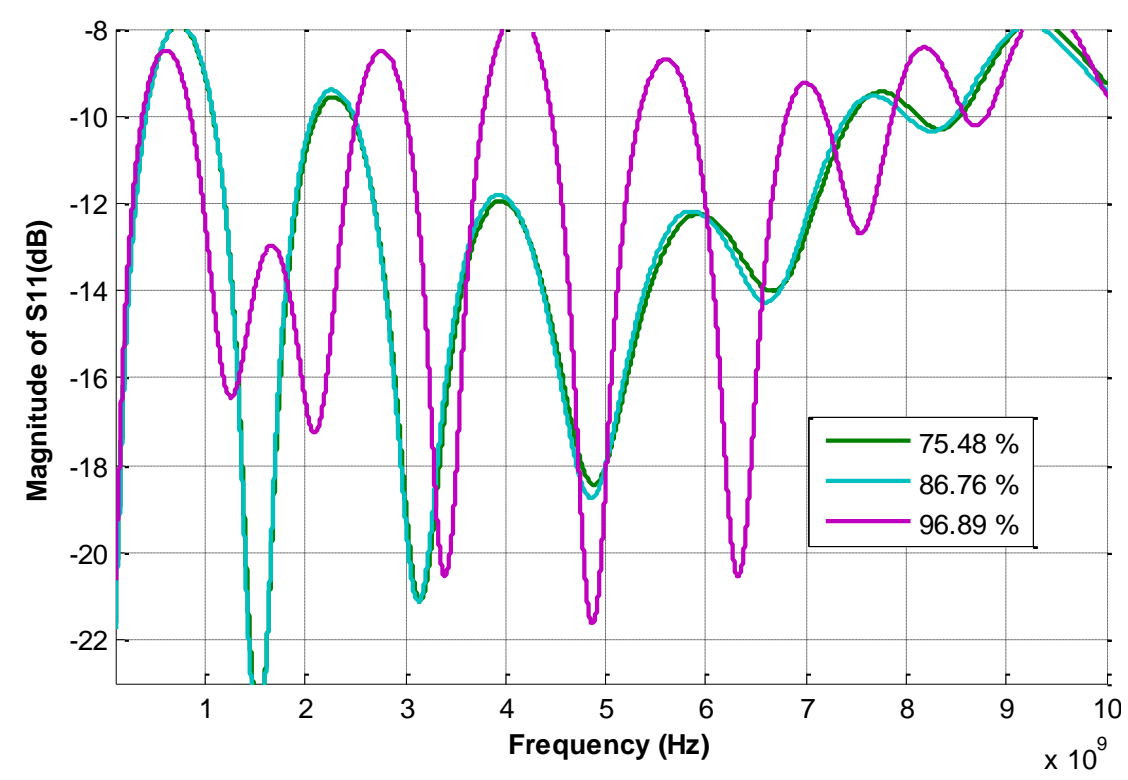

Fig. 7. Measured magnitude of $\mathrm{S}_{11}$.

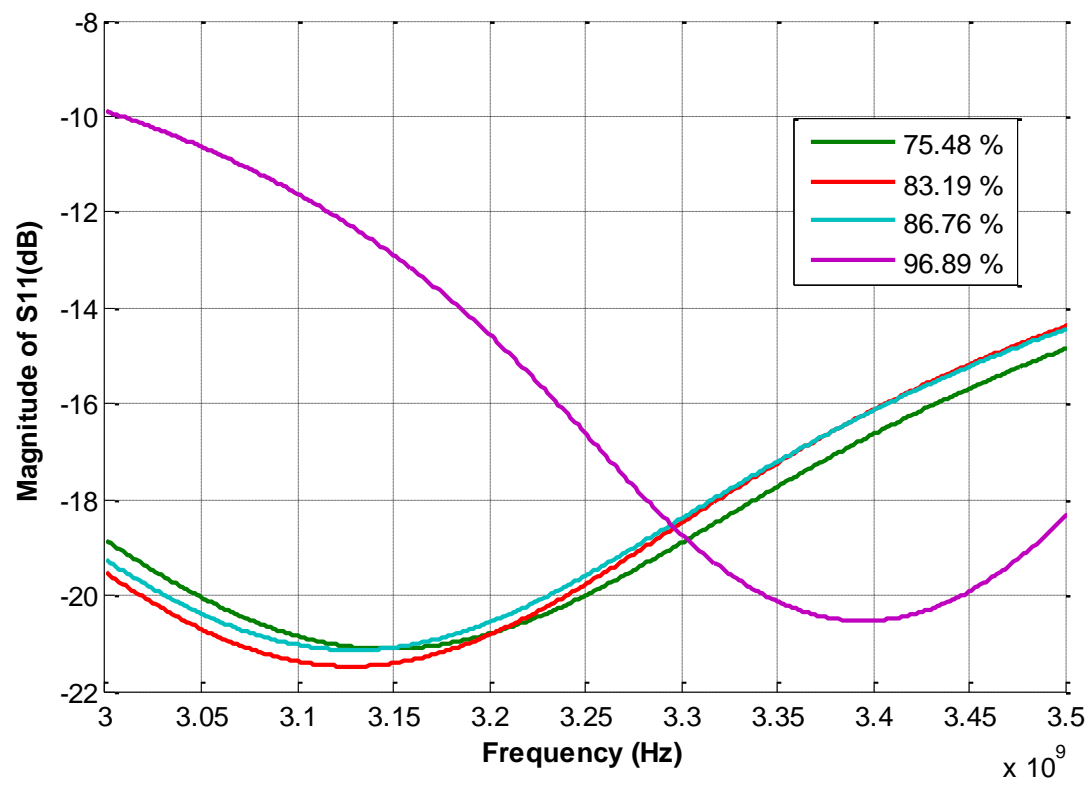

Fig. 8. Measured magnitude of $\mathrm{S}_{11}$ between 3 to $3.5 \mathrm{GHz}$ 


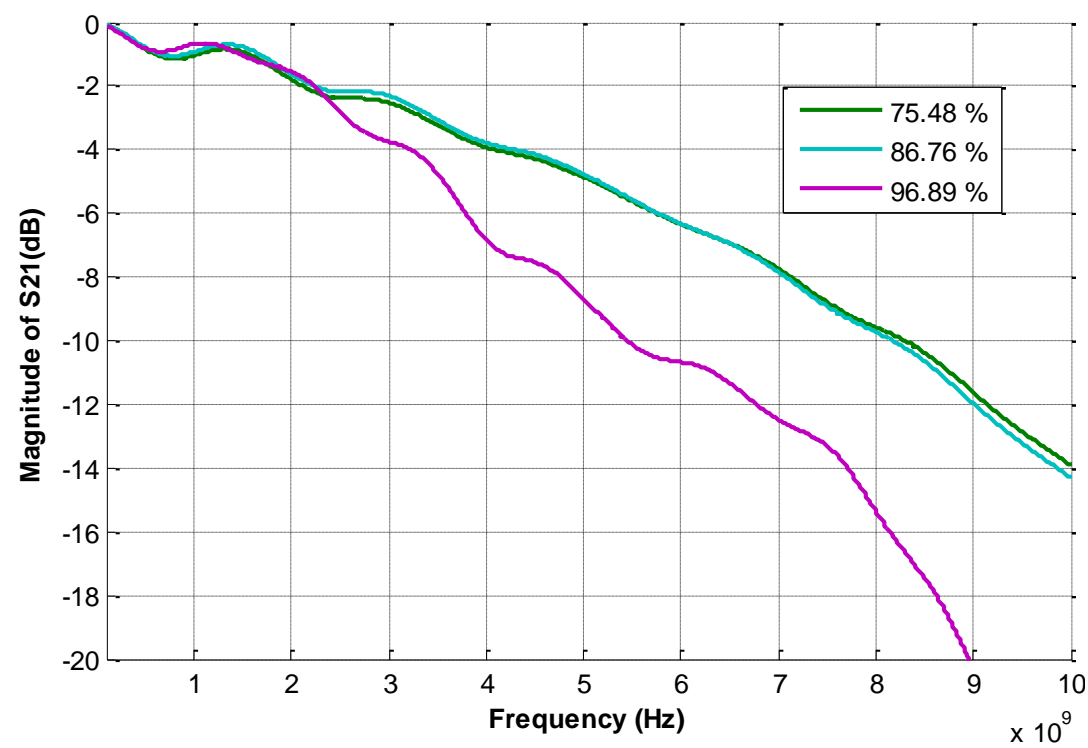

Fig. 9. Measured magnitude of $\mathrm{S}_{21}$.

The real parts of transmission coefficient for samples with 75.48, 86.76 and 96.89 percent purification are shown in Fig. 10. The results confirm compatibility with Eq. (11) and are similar to the simulation results in Fig. 6. The amplitude is obviously related to the dielectric loss and the distance between zerocrossings is related to the dielectric constant. Fig. 11 and 12 show the real and imaginary parts of complex relative permittivity values of LUT which are calculated by Eq. (11) to (17).

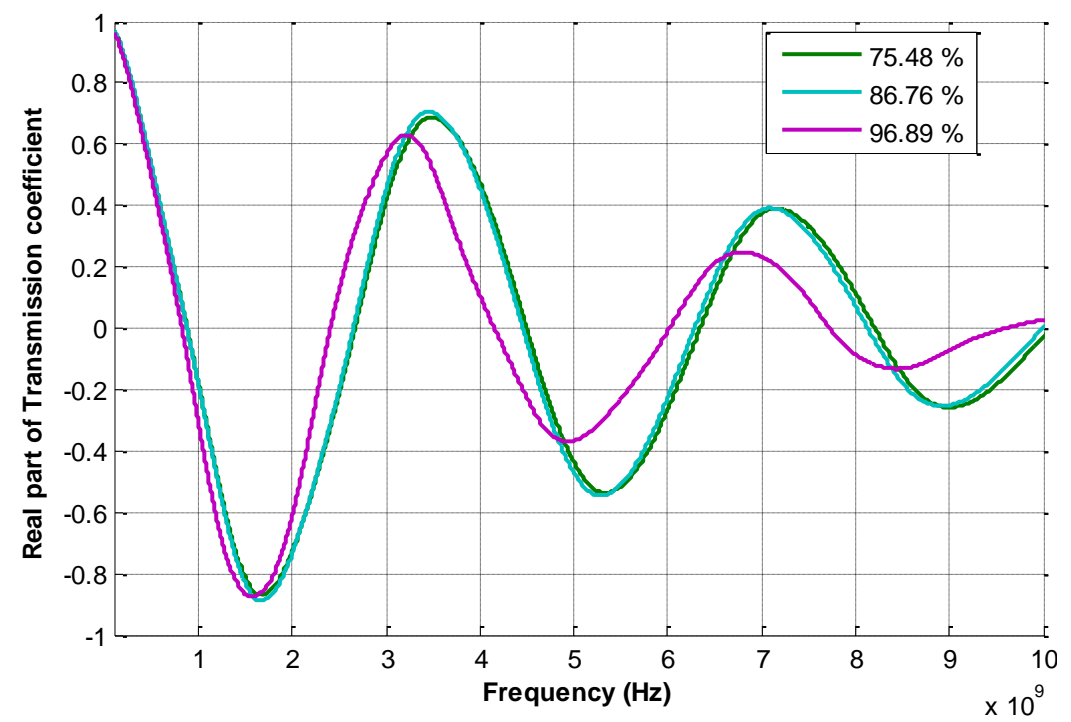

Fig. 10. Real part of transmission coefficient of the measurement result.

Calculated real and imaginary parts of complex relative permittivity are shown in Fig. 11 and 12 , respectively. Purification of biodiesel can be investigated using complex permittivity values, especially in the frequency range between 2 to $4 \mathrm{GHz}$. 


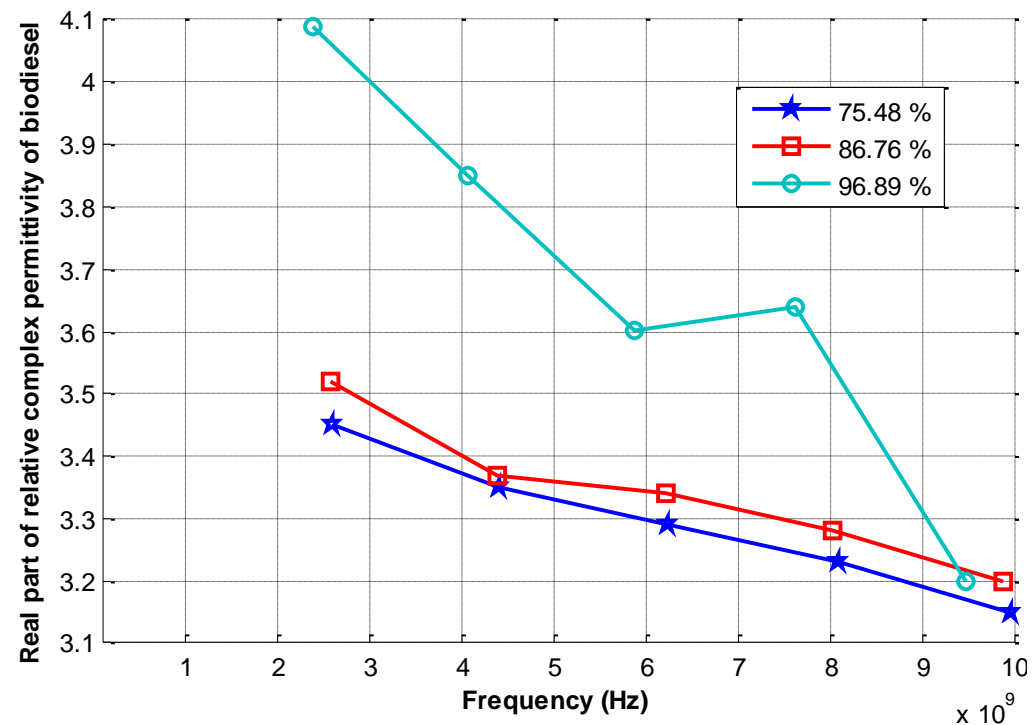

Fig. 11. Real part of complex relative permittivity of biodiesel samples with different purification.

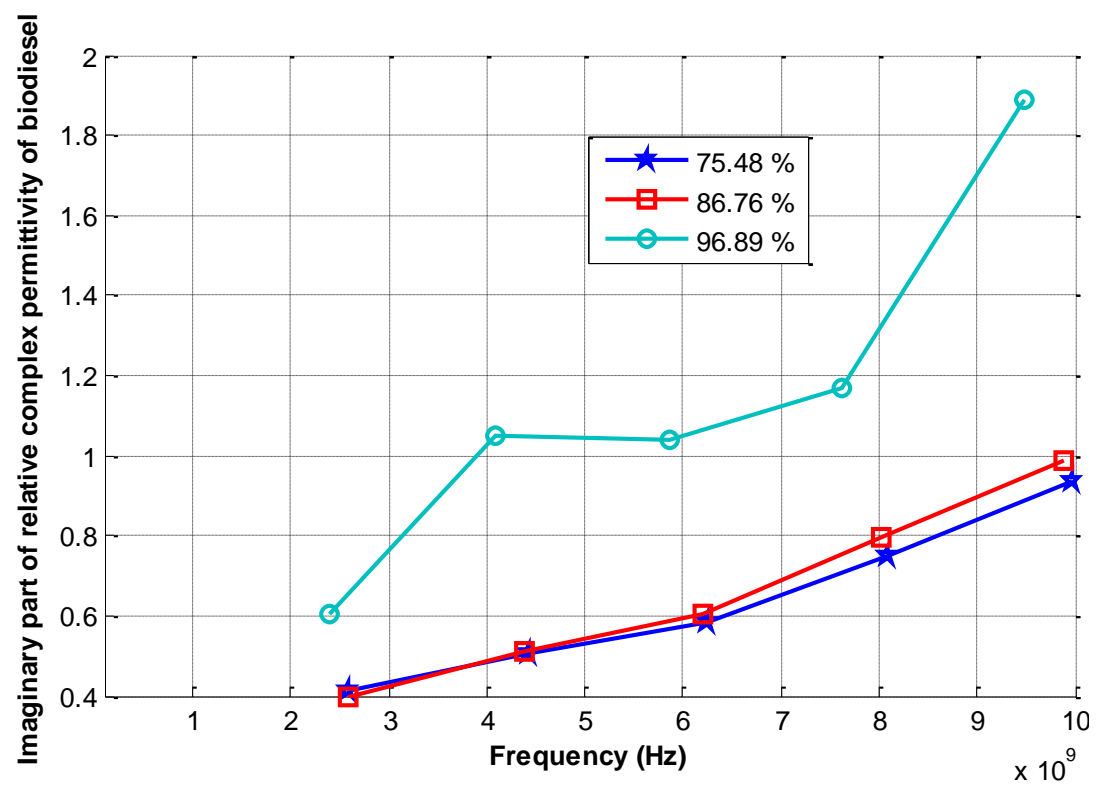

Fig. 12. Imaginary part of complex relative permittivity of biodiesel samples with different purification.

Considering the relative complex permittivity of the samples, the purification level is higher with higher real part of the complex permittivity. The same trend is also observed for the imaginary part. From the results shown in Fig. 11 and 12, the purification levels of $75.48 \%$ and $86.76 \%$ show a slight difference of the complex permittivity. In case of the sample with $96.89 \%$ purification, the complex permittivity is significantly different to the sample with $86.76 \%$. In order to obtain more information about the relationship between the permittivity and the purification level, measurements with more samples are required e.g. for the purification level between $86.76 \%$ and $96.89 \%$ as well as for values of lower than $75.48 \%$

In addition, IFTs of the transmission coefficients for all cases were calculated. The IFT of the transmission coefficient consists of real $(\operatorname{Re}\{\operatorname{IFT}\})$ and imaginary $(\operatorname{Im}\{\operatorname{IFT}\})$ parts as shown in Fig. 13 and 14, respectively. The zero crossing point t of $\operatorname{Im}\{\mathrm{IFT}\}$ is directly related to the dielectric constant of the LUT (see Eq. 21) and the power stored in the material. The power dissipation in the material is represented by the peak amplitude of $\operatorname{Re}\{I F T\}$ at the time point $t$. 


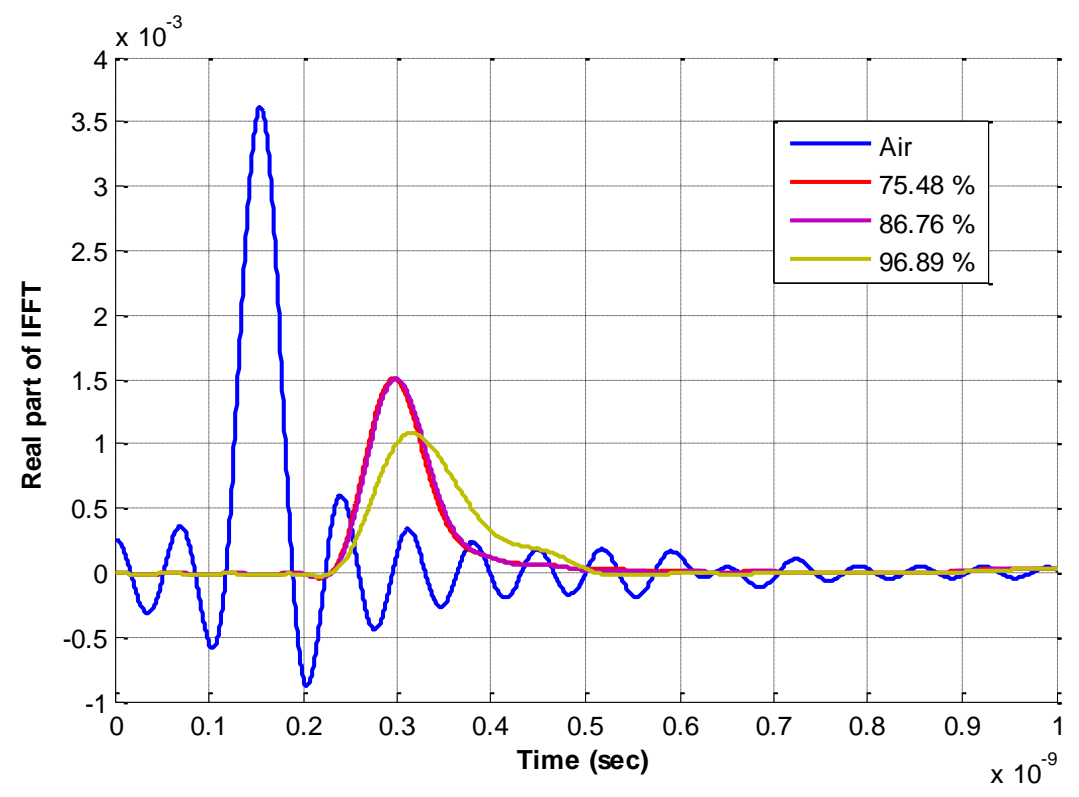

Fig. 13. $\operatorname{Re}\{\mathrm{IFT}\}$ of real part of the transmission coefficient.

For sensing of biodiesel purification, the zero crossing point $\mathrm{t}$ can be used. High value of $\mathrm{t}$ represents high power storage, high dielectric constant and high purification (see Fig. 16). From this result, we can conclude that high-purity biodiesel has normally high dielectric constant. In case of $\operatorname{Im}\{\mathrm{IFT}\}$ the purifications of 75 and 86 do not show significant difference of the peak values (see Fig. 15). Therefore, this parameter is not appropriate for sensing of biodiesel purification. The values of $\mathrm{t}$ and peak magnitude of Re $\{$ IFT $\}$ are shown for all investigated biodiesel samples with different purification levels in Table 1 .

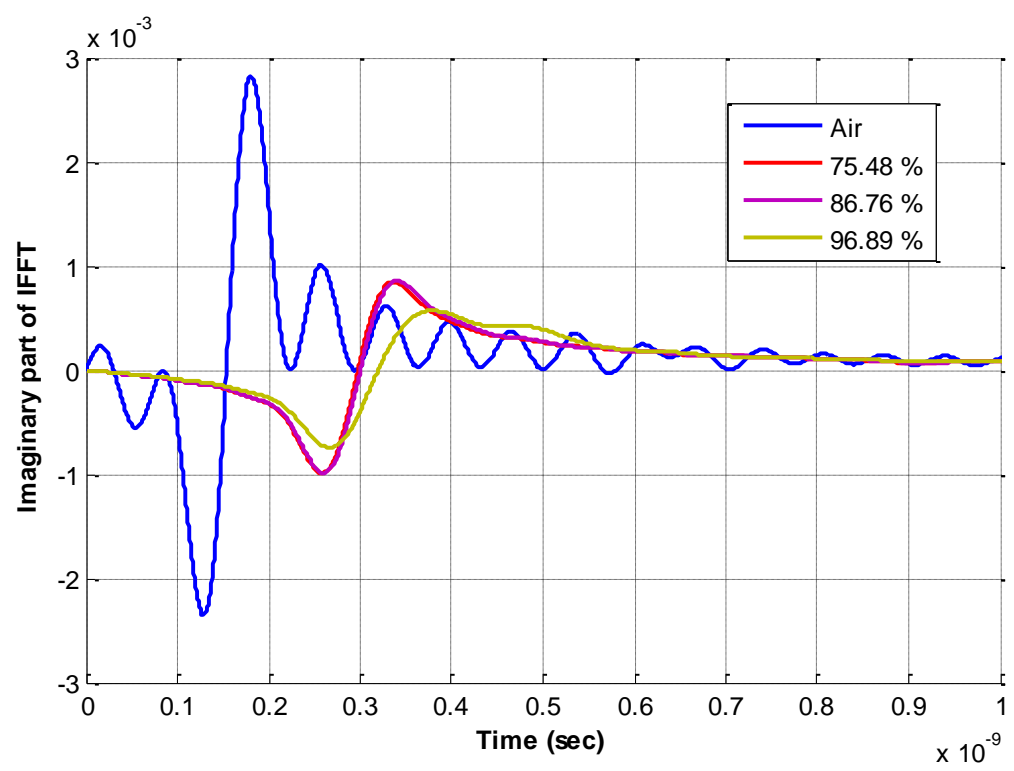

Fig. 14. $\operatorname{Im}\{\mathrm{IFT}\}$ of real part of the transmission coefficient. 


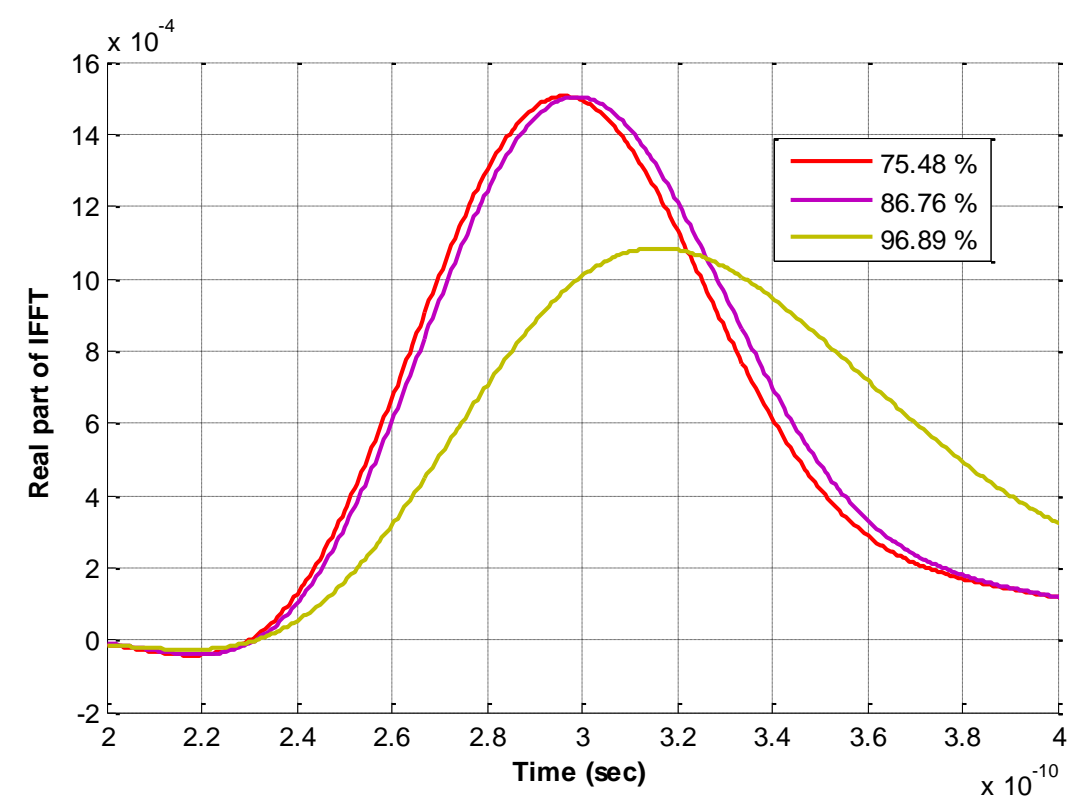

Fig.15. $\operatorname{Re}\{\mathrm{IFT}\}$ with different biodiesel samples between $2 \times 10^{-10}$ to $6 \times 10^{-10} \mathrm{sec}$.

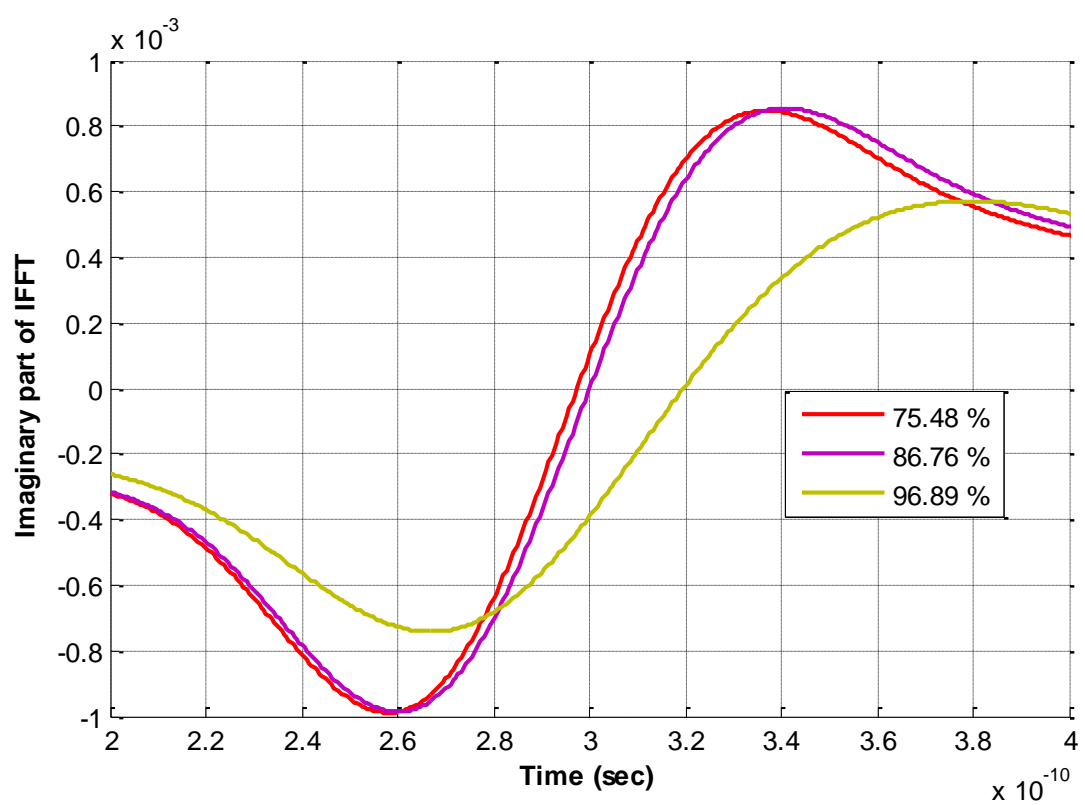

Fig. 16. $\operatorname{Im}\{\mathrm{IFT}\}$ with different biodiesel samples in between 0.2 to $0.6 \mathrm{nsec}$.

Table 1. Zero-crossing point t of $\operatorname{Im}\{$ IFT $\}$ and corresponding peak amplitude of $\operatorname{Re}\{\operatorname{IFT}\}$ in case of different purification levels of investigated biodiesel samples.

\begin{tabular}{ccc}
\hline $\begin{array}{c}\text { Purification of } \\
\text { biodiesel sample (\%) }\end{array}$ & $\begin{array}{c}\text { Zero-crossing } \\
\text { point t of Im\{IFT }\}\end{array}$ & $\begin{array}{c}\text { Peak } \\
\text { amplitude of } \\
\text { Re }\{\text { IFT }\}\end{array}$ \\
\hline 75.48 & $2.973 \times 10^{-10}$ & $1.504 \times 10^{-3}$ \\
83.19 & $2.998 \times 10^{-10}$ & $1.506 \times 10^{-3}$ \\
86.76 & $3.000 \times 10^{-10}$ & $1.502 \times 10^{-3}$ \\
96.89 & $3.195 \times 10^{-10}$ & $1.081 \times 10^{-3}$ \\
\hline
\end{tabular}




\section{Conclusions}

The purification of biodiesel is directly related to its complex permittivity which can be calculated from the transmission coefficient. The real part of complex permittivity describes the power stored in the material which depends on the frequency. In a frequency range between 2 to $4 \mathrm{GHz}$, the level of purification can be mapped to the real part of complex permittivity. In the same frequency range, the level of purification can be characterized by power dissipation in the material which is described by the imaginary part of complex permittivity.

The inverse Fourier transform (IFT) of real part of the transmission coefficient is another technique for biodiesel purification sensing. The results are in complex form which describes power stored and dissipated in the material in time domain.

The real part representing the energy dissipated in the material cannot clearly differentiate the purification level of $75.48,83.19$ and 86.76 percent. The parameter which is more appropriate for biodiesel purification sensing is the zero-crossing point in time domain of imaginary part. This parameter is directly related to energy stored in the material and the dielectric constant. High purity biodiesel sample shows high dielectric constant and large zero-crossing time of the imaginary part.

\section{Acknowledgement}

This work was financially supported by the Thailand Research Fund (TRF) under the industrial RGJ Ph.D. program.

\section{References}

[1] M. F. Yahaya, I. Demshemino, I. Nwadike, O. P. Sylvester, and L. N. Okoro, "A review on the chromatographic analysis of biodiesel," International Journal of Education and Research, vol. 1, no. 8, Aug. 2013.

[2] T. Ruppel and T. Huybrighs, "Gas chromatography," PerkinElmer, Inc. Shelton, CT, USA.

[3] J. D. McCurry, "GC analysis of total fatty acid methyl esters (FAME) and methyl linolenate in biodiesel using the revised EN14103:2011 method," Agilent Technologies Inc., USA.

[4] M. U. Afsar, J. R. Birch, R. N. Clarke, and G. W. Chantry, "The measurement of properties of material," in Proceeding of the IEEE, vol. 74, pp. 183-199, 1986.

[5] G. Banhegyi, "Dielectric spectroscopy," in Characteruzation of Composite Material. Boston: ButterworthHeinemann, 1994.

[6] J. Krupka, "Frequency domain complex permittivity measuring at microwave frequencies," Measurement Science and Technology, vol. 17, pp. 55-70, 2006.

[7] L. F. Chen, C. K. Ong, C. P. Neo, V. V. Varadan, and V. K. Varadan, "Overview of the microwave for characterization of electromagnetic material," in Microwave Electronics Measurement and Material Characterization. John Wiley \& Sons, Ltd., 2004.

[8] A. Kumar and S. Sharma, "Measurement of dielectric constant and loss factor of the dielectric material at microwave frequencies," Progress In Electromagnetics Research PIER, vol. 69, pp. 47-54, 2007.

[9] M. S. Venkatesh and G. S. V. Raghavan, "An overview of dielectric properties measuring techniques," Canadian Biosystem Engineering, vol. 47, pp. 7.15-7.30, 2005.

[10] O. V. Tereshchenko, F. J. K. Buesink, and F. B. J. Leferink, "An overview of the techniques for measuring the dielectric properties of materials," in Proc. General Assembly and Scientific Symposium 30th URSI, Istanbul, 2011, pp. 1-4.

[11] S. Robert and A. von Hippel, "A new method for measuring dielectric constant and loss in the range of centimeter," Journal of Applied Physics, vol. 17, pp. 610-616, 1946.

[12] Y. Kuriyama, N. Ueda, A. Nishikata, K. Fukunaga, S. Watanabe, and Y. Yamanaka, "Liquid materials complex permittivity measurement using a rectangular waveguide and dielectric tube at 800 and 900 MHz band", in Proc. International Symposium on Electromagnetic Compatibility, Sendai, Japan, 2004, vol. 2, pp. 645-648.

[13] Y. Wang and M. N. Afsar, "Measurement of complex permittivity of liquid using waveguide techniques," Progress In Electromagnetic Research, vol. 42, pp. 131-142, 2003. 
[14] J. P. Grant, R. N. Clark, G. T. Symm, and N. M. Spyrou, "A critical study of the open-end coaxial line sensor technique for RF and microwave complex permittivity measurement," Journal of physics: Electronics and Scientific Instrument, vol. 22, pp. 757-770, 1989.

[15] N. I. Sheen and I. M. Woodhead, "An open-ended coaxial probe for broad-band permittivity measurement of agricultural product," Jourlnal of Agricultural Engineering Research, vol. 74, pp. 193-202, 1999.

[16] H. Kawabata and Y. Kobayashi, "Accurate measurements of complex permittivity of liquid based on a TM010 mode cylindrical cavity method," in Proceedings of the 35th European Microwave Conference, EuMC17, pp. 369-372, 2005.

[17] H. Kawabata, H. Tanpo, and Y. Kobayashi, "An improvement of the perturbation method using a TM010 mode cylindrical cavity," IEICE Trans. Electron., vol. E86-C, no. 12, pp. 2371-2378, 2003.

[18] P. Kanpan, E. Khansalee, N. Puangngernmak, and S. Chalermwisutkul, "TM010 mode cylindrical cavity for complex permittivity measurement of liquid using field analysis technique," in Proc. Electrical Engineering/Electronics, Computer, Telecommunications and Information Technology Conference, ECTI-CON 9, pp. $1-4,2012$.

[19] S. Ramo, J. R. Whinnery, and T. Van Duzer, Field and Wave in Communication Electronic, 3rd ed. New York: John Wiley \& Son, Inc.

[20] B. C. Wadell, Transmission Line Design Handbook. Norwood, MA: Artech House, Inc., 1991.

[21] W. J. Ellison, K. Lamkaouchi, and J. M. Moreau, "Water: A dielectric reference," J. Molec. Liquids, vol. 68, p. 233, 1996. 
